\title{
JNK signaling is required for the MIP-1 $\alpha$-associated regulation of Kupffer cells in the heat stroke response
}

\author{
XIAO-JUAN CHEN ${ }^{1 *}$, ZHONG-ZHI TANG ${ }^{1 *}$, GUO-GUO ZHU ${ }^{1}$, QING CHENG $^{1}$, \\ WEN-KAI ZHANG ${ }^{1}$, HUI-MIN LI ${ }^{1}$, WEI FU ${ }^{1}$ and QI-PING LU ${ }^{2}$ \\ Departments of ${ }^{1}$ Emergency and ${ }^{2}$ General Surgery, Wuhan General Hospital of \\ Guangzhou Military Command, Wuhan, Hubei 430070, P.R. China
}

Received March 17, 2016; Accepted March 6, 2017

DOI: $10.3892 / \mathrm{mmr} .2017 .6922$

\begin{abstract}
Severe heat stroke (HS) consists of extreme hyperthermia with thermoregulatory failure, leading to high morbidity and mortality. Liver injury is a complication of HS that is associated with inflammatory responses and Kupffer cells (KCs), which are resident macrophages in the liver that serve as a major source of inflammatory cytokines; however, the association and the underlying mechanisms of $\mathrm{KC}$ functions in HS-induced endotoxemia and inflammation require an improved understanding. The important chemokine macrophage inflammatory protein- $1 \alpha$ (MIP- $1 \alpha$ ) increases inflammatory responses and the secretion of inflammatory molecules from KCs, including tumor necrosis factor- $\alpha$, interleukin (IL)-1 $\beta$ and IL-6. In addition, the activation of c-Jun N-terminal kinase (JNK) signaling is responsible for the development of liver inflammation. Therefore, HS animal and cell models were constructed in order to investigate the pathways involved in the HS-induced dysfunction of KCs. The results of the present study suggest that JNK may be involved in the MIP-1 $\alpha$-associated pathogenesis of KCs in HS injury.
\end{abstract}

\section{Introduction}

Heat stroke (HS) is a life-threatening disease with high morbidity and mortality that is characterized by extreme hyperthermia $\left(>40^{\circ} \mathrm{C}\right)$ with thermoregulatory failure (1). Since heatwaves are responsible for HS injury and mortality in various parts of the world $(2,3)$, there is a growing concern regarding the prevention and treatment of HS, particularly

Correspondence to: Dr Qi-Ping Lu, Department of General Surgery, Wuhan General Hospital of Guangzhou Military Command, 627 Wuluo Road, Wuchang, Wuhan, Hubei 430070, P.R. China

E-mail: qplu16@sina.com

*Contributed equally

Key words: heat stroke, Kupffer cell, macrophage inflammatory protein $1 \alpha$, c-Jun $\mathrm{N}$-terminal kinase during military training in China $(4,5)$. Accumulating evidence suggests that endotoxins and cytokines are implicated in the pathogenesis of HS (6). Inflammatory cytokines are highly elevated in some HS patients and in animal models, and these levels correlate with organ failure and a fatal outcome (7). The liver, a major organ of immunity that produces and responds to cytokines during inflammation, is notably damaged in HS (8). The liver serves an important role in heat stress responses through the production of cytokines and, particularly, in the clearance of intestinal-derived endotoxins produced during hyperpyrexia, including lipopolysaccharide (LPS) (9), which is associated with the regulation of Kupffer cells (KCs). KCs are the primary source of intrahepatic inflammatory cytokines (10) and the primary cause of increased LPS concentrations (11).

KCs account for $80-90 \%$ of total resident macrophages in vivo (12). KCs reside in the liver and remove immune complexes and intestinal bacterial products from the blood, which is essential for immunity against various pathologies (13). KCs are critical in the clearance of gut-derived endotoxins by phagocytosis. Under normal conditions, KCs are involved in the interruption of the formation of endotoxins by phagocytosis and the simultaneous release of cytokines. Under conditions of ischemia-reperfusion, hemorrhagic shock and hemorrhagic pancreatitis, phagocytotic function of KCs is weakened by endotoxemia; however, their secretory function enhances inflammation, which results in multiple organ failure (14). A previous study has reported that liver injury in rats with $\mathrm{HS}$ is associated with the increased secretion of tumor necrosis factor (TNF)- $\alpha$, interleukin (IL)- $1 \beta$ and IL- 6 by KCs (5). Previous evidence suggests that the macrophage-derived macrophage inflammatory protein-1 $\alpha$ (MIP-1 $1 \alpha)$ stimulates the secretion of TNF- $\alpha$, IL- $1 \beta$, and IL- 6 by peritoneal macrophages (15). The association between MIP-1 $\alpha$ and macrophages indicates that the expression of MIP-1 $\alpha$ influences the alteration in the concentration of inflammatory cytokines secreted by KCs under heat stress.

MIP- $1 \alpha$ is an important chemokine that activates immunocytes, promotes their chemotaxis, regulates the synthesis of cytokines and is involved in acute or mild inflammation (16). MIP-1 $\alpha$ is produced by immune cells, including macrophages, lymphocytes, neutrophils and dendritic cells (17). As the largest group of macrophages in the human body, KCs are the major source of MIP-1 $1 \alpha$ in the liver. In 
the MIP-1 $\alpha$ knock-out rat model, the levels of MIP-1 $\alpha$ and the levels of cytokines, including TNF- $\alpha$, IL-1 $\beta$ and IL-6, secreted by $\mathrm{KCs}$ are reduced compared with normal rats, and these alterations result in the downregulation of inflammatory responses (17). A previous study on acute inflammatory liver injury demonstrated that MIP- $1 \alpha$ secreted by $\mathrm{KCs}$ is increased, and anti-MIP-1 $\alpha$ functions to decrease the inflammatory responses as well as the mortality rate of the rats (18). A previous study using a liver ischemia-reperfusion injury model demonstrated that MIP- $1 \alpha$ secreted by KCs is responsible for the inflammation associated with multiple organ failure (19). Therefore, MIP-1 $\alpha$ serves an important role in $\mathrm{KC}$-associated inflammatory responses.

As MIP-1 $\alpha$ elevates inflammatory responses, it ensures that increased levels of inflammation are more serious in HS-induced liver injury. In order to identify the signaling pathways that mediate MIP- $1 \alpha$-associated inflammation in $\mathrm{KCs}$, c-Jun N-terminal kinase (JNK) signaling was investigated, as previous studies have demonstrated that activation of the JNK signaling pathway contributes to the development of liver inflammation (20). JNK, which is activated by various stimuli, including cytokines, serves a role in cell responses, inflammation and apoptosis (17). Previous research demonstrates that the JNK signaling pathway is potentially activated in trauma, stress and ischemia-reperfusion (21). A previous study have demonstrated that JNK signaling is partially responsible for the activation of macrophages and macrophage-induced renal injury (22). In addition, the activation of JNK signaling stimulates the production of pro-inflammatory factors, including TNF- $\alpha$ and IL-1, which lead to further exacerbation of the inflammatory response (23). It was hypothesized that $\mathrm{KC}$-secreted MIP-1 $\alpha$ promotes inflammation through the JNK signaling pathway during HS. In the present study, HS animal and cell models were constructed to detect changes in KCs and the association with MIP-1 $\alpha$ and the JNK signaling pathway.

\section{Materials and methods}

Experimental animals. The experiments were performed in 40 adult male Wistar rats weighing 250-300 g and aged 10-12 weeks old, obtained from the Animal Resource Center of Hubei Provincial Academy of Preventive Medicine (Wuhan, China). The animals were housed at a temperature of $25^{\circ} \mathrm{C}$ and were given free access to food and water in the specific pathogen-free facility at Wuhan General Hospital of Guangzhou Command (Wuhan, China). All of the animal studies were approved by the Animal Ethics Committee of Wuhan General Hospital of Guangzhou Command in accordance with the Guide for the Care and Use of Laboratory Animals of the State Scientific and Technological Commission of China.

The animals were randomly assigned to two groups. The experimental group consisted of rats that were placed in a warm blanket of the instrument for maintaining animal body temperature (SS-20-2; Anhui Huaibei Zhenghua Biological Apparatus Co., Ltd., Anhui, China) at $40^{\circ} \mathrm{C}$ throughout the experiment and was correspondingly named the HS group. An intraperitoneal injection of sodium pentobarbital $(50 \mathrm{mg} / \mathrm{kg}$ body weight) was administered to anesthetize the animals.
Induction of heat stress. The rats in the experimental group were placed in a folded heating blanket, with the temperature set to $40^{\circ} \mathrm{C}$. A towel was placed between the animals and the warm blanket to avoid burns. The core temperature (represented by the rectal temperature) was monitored by the PowerLab Data Acquisition and Analysis System (AD Instruments, Shanghai, China) every $5 \mathrm{~min}$. The point at which the systolic blood pressure (represented by the caudal artery systolic pressure) decreased from its peak value was taken as the onset of HS. The rats were removed immediately from the heating blanket and returned to room temperature $\left(25^{\circ} \mathrm{C}\right)$.

Isolation of KCs. The liver was perfused with physiological saline containing $0.01 \%$ EDTA through the portal vein for $\sim 25$ min until the liver tissue was completely softened. The liver was removed, crushed using scissors and digested into liquid, which was filtered through a 200 -mesh strainer into a $50 \mathrm{ml}$ centrifuge tube. The filtrate was washed thoroughly in RPMI-1640 (Hyclone; GE Healthcare Life Sciences, Logan, UT, USA) at $2,500 \mathrm{x} \mathrm{g}$ for $5 \mathrm{~min}$ at $4^{\circ} \mathrm{C}$ and suspended in $12 \mathrm{ml}$ RPMI-1640. A $12 \mathrm{ml}$ cell suspension was placed on $15 \mathrm{ml} \mathrm{50 \%} \mathrm{Percoll} \mathrm{solution} \mathrm{(Pharmacia;} \mathrm{GE} \mathrm{Healthcare} \mathrm{Life}$ Sciences, Little Chalfont, UK) and $20 \mathrm{ml} \mathrm{25 \%} \mathrm{Percoll} \mathrm{solu-}$ tion, and the mixture was centrifuged at $2500 \mathrm{rpm}$ for $20 \mathrm{~min}$. The cells between the layers of $25 \%$ Percoll solution and $50 \%$ Percoll were slowly extracted and resuspended in RPMI-1640 containing 10\% fetal bovine serum (Gibco; Thermo Fisher Scientific, Inc., Waltham, MA, USA), penicillin (100 U/ml) and streptomycin $(100 \mu \mathrm{g} / \mathrm{ml})$. The cell suspensions were plated on $10-\mathrm{cm}$ culture dishes and placed in a humidified incubator at $37^{\circ} \mathrm{C}$, containing $5 \% \mathrm{CO}_{2}$. After $6 \mathrm{~h}$, the cell supernatants were discarded, and the cells were washed three times with PBS at $37^{\circ} \mathrm{C}$. In total $>95 \%$ of the adherent cells were KCs, as determined by examination of cluster of differentiation (CD)163 staining, and the cell viability $>90 \%$, as demonstrated through trypan blue exclusion analysis. To explore whether the JNK signaling pathway was associated with the regulation of phagocytic and secretory functions of KCs during HS, a JNK inhibitor (SP600125; Wuhan Myhalic Biotechnology Co., Ltd., Wuhan, China) was used to treat cultured KCs. KCs were cultured for $24 \mathrm{~h}$, and then treated with $50 \mu \mathrm{M}$ SP600125 for the indicated periods of time.

Measurement of phagocytic function in vivo and in vitro. The phagocytic activity of KCs in each group were determined following HS termination. In vivo, the caudal vein was injected with a 1:10 dilution of India ink in $0.9 \%$ saline $(0.25 \mathrm{ml} / 100 \mathrm{~g})$. The animal was sacrificed following the India ink injection, and the liver was processed and stained with eosin. The consumption of carbon by KCs was observed with a microscope. LPS concentrations were detected in the peripheral blood using a ToxinSensor ${ }^{\mathrm{TM}}$ Chromogenic LAL Endotoxin Assay kit (GenScript Corporation, Piscataway, NJ, USA) according to the manufacturer's protocol. Orbital blood was collected following the injection of diluted India ink, and the phagocytic index $\mathrm{K}$ value was calculated subsequent to the absorbance being measured (24). In vitro, isolated KCs were plated in a 96-well plate at a density of $5 \times 10^{6}$ cells $/ \mathrm{ml}$ and incubated overnight. pHrodo $^{\text {TM }}$ Escherichia coli BioParticle-conjugated particles 
(Molecular Probes; Thermo Fisher Scientific, Inc.), dissolved in RPMI-1640 at a concentration of $1 \mathrm{mg} / \mathrm{ml}$, were added to each well. The procedure was performed using the $\mathrm{pHrodo}^{\mathrm{TM}}$ E. coli BioParticles ${ }^{\circledR}$ Phagocytosis kit for Flow Cytometry (Invitrogen; Thermo Fisher Scientific, Inc.) following the manufacturer's protocol. The cells were resuspended in $200 \mu \mathrm{l}$ staining buffer and analyzed using an LSRII flow cytometer (BD Biosciences, Franklin Lakes, NJ, USA). Data analysis was conducted using the accompanying FACSDiva v8.0 and FCAP Array v3.0 software (BD Biosciences). Interferon (IFN) $-\gamma$ (RA20684), IL-1 $\beta$ (RA20020), TNF- $\alpha$ (RA20035) and MIP-1 $\alpha$ (RA20996) concentrations in the medium of cultured KCs and the peripheral blood, were detected using ELISAs (Bio-swamp Life Science Lab, Wuhan, China) in the HS and control groups.

Silencing of MIP-1 $\alpha$. The small interfering RNA (siRNA) targeting rat MIP-1 $\alpha$ (siMIP-1 $\alpha$; 5'-CAGCGCCAUAUG GAGCUGAC-3') was purchased from Thermo Fisher Scientific, Inc. KCs were transfected with siRNAs using Lipofectamine ${ }^{\circledR} 2000$ (Invitrogen; Thermo Fisher Scientific, Inc.) according to the manufacturer's protocol. Assays were performed $48 \mathrm{~h}$ following transfection.

Immunofluorescence. Liver tissues were fixed in formalin and blocked in $10 \%$ normal goat serum (Wuhan Boster Biological Technology, Ltd., Wuhan, China) for $30 \mathrm{~min}$ at $37^{\circ} \mathrm{C}$. The tissues were incubated with a primary antibody against CD163 (1:100; sc-18796; Santa Cruz Biotechnology, Inc., Dallas, TX, USA) overnight at $4^{\circ} \mathrm{C}$ and amplified with rabbit anti-goat IgG secondary antibody (1:1,000; ab150141; Abcam, Cambridge, England) conjugated to Alexa Fluor 488 for $1 \mathrm{~h}$ at $37^{\circ} \mathrm{C}$. For co-localization with CD163, sections were co-stained overnight with a primary antibody against p-JNK (1:100; sc-293136) or MIP-1 $\alpha$ (1:100; sc-365691) (both from Santa Cruz Biotechnology, Inc.) overnight at $4^{\circ} \mathrm{C}$ and amplified with goat anti-mouse $\operatorname{IgG}$ secondary antibody (1:500; ab175473; Abcam) conjugated to Alexa Fluor 568 for $1 \mathrm{~h}$ at $37^{\circ} \mathrm{C}$. Prior to observation, DAPI was used to label the nuclei. Images were acquired using a fluorescence microscope (OLYMPUS BX51; Olympus Corporation, Tokyo, Japan) with appropriate filters. The images are presented as single-color stains of green and red to exhibit the localization of the two markers in the cells. Merged images are presented below the images with single-color stains. The two markers that were co-expressed in cells at a similar location are often observed in yellow.

Immunoblotting. Following the different treatments, the cells or livers were harvested, washed three times with PBS and lysed in radioimmunoprecipitation buffer [50 mM Tris- $\mathrm{HCl}(\mathrm{pH} 7.4)$, $150 \mathrm{mM} \mathrm{NaCl}, 1 \% \mathrm{NP}-40,0.5 \%$ sodium deoxycholate, $0.1 \%$ SDS, $1 \mathrm{mM}$ EDTA, $30 \mu \mathrm{g} / \mathrm{ml}$ aprotinin, $50 \mu \mathrm{g} / \mathrm{ml}$ leupeptin and $1 \mathrm{mM}$ phenylmethane sulfonyl fluoride] on ice for $30 \mathrm{~min}$. The cell lysates were centrifuged at $12,000 \mathrm{x} \mathrm{g}$ and $4^{\circ} \mathrm{C}$ for $10 \mathrm{~min}$, and the supernatant was collected. Protein concentrations were measured using a Bicinchoninic Acid Protein Assay kit (P0010S; Beyotime Institute of Biotechnology, Shanghai, China). Protein was loaded at a concentration of $30 \mu \mathrm{g}$ per lane and separated by $10 \%$ SDS-PAGE. Following electrophoresis, the proteins were transferred onto polyvinyl difluoride membranes (Merck KGaA, Darmstadt, Germany), incubated with $5 \%$ non-fat milk for $1 \mathrm{~h}$ and then incubated overnight at $4^{\circ} \mathrm{C}$ with the primary antibodies. The primary antibodies were as follows: Anti-MIP-1a antibody (1:200; sc-365691; Santa Cruz Biotechnology, Inc.) and anti-phospho-JNK (p-JNK) antibody (1:200; sc-293136; Santa Cruz Biotechnology, Inc.). The protein loading was normalized with anti- $\beta$-actin antibody (1:200; sc-58673; Santa Cruz Biotechnology, Inc.). An HRP-conjugated secondary antibody (1:5,000; BM3895; Boster Biological Technology, Pleasanton, CA, USA) was used, and the signals were detected using an ECL Super Signal West Pico Trial kit (Thermo Fisher Scientific, Inc.).

Statistical analysis. Results are expressed as the mean \pm standard deviation. All experimental data were analyzed using one-way analysis of variance followed by the least significant difference t-test. $\mathrm{P}<0.05$ was considered to indicate a statistically significant difference. All analyses were performed using SPSS software (version 19.0; IBM SPSS, Armonk, NY, USA).

\section{Results}

HS inhibits the phagocytic activity of KCs. To determine whether HS is responsible for changes in the phagocytic function of KCs, latex beads and a pHrodo E. coli BioParticles Phagocytosis kit for Flow Cytometry was used to detect the phagocytic activity of cultured rat KCs in each group. The phagocytic activity of KCs was significantly decreased in the HS group compared with the control group, as presented in Fig. 1A-C $(\mathrm{P}<0.05)$.

In order to estimate the phagocytic function of $\mathrm{KCs}$ in vivo and further confirm that increased temperatures affect the phagocytic function of KCs, the livers of each group were stained with eosin, and the consumption of carbon by the KCs was observed microscopically subsequent to the caudal vein being injected with diluted India ink (Fig. 1D). As presented in Fig. 1E, the phagocytic activity of KCs exhibited a trend similar to that observed in vitro $(\mathrm{P}<0.05)$. KCs help protect the intestines from attack by bacteria and endotoxins via phagocytosis and removal from the blood. In order to further analyze the phagocytic function of KCs in vivo, LPS concentrations were detected in the peripheral blood using a ToxinSensor ${ }^{\mathrm{TM}}$ Chromogenic LAL Endotoxin Assay kit. As presented in Fig. 1F, the LPS concentration was increased at HS onset and continued to increase to a high level compared with that in the control group $(\mathrm{P}<0.05)$, indicating that $\mathrm{KCs}$ failed to eliminate endotoxins from the blood. The results of the present study suggest that the phagocytic activity of $\mathrm{KCs}$ is reduced in $\mathrm{HS}$ conditions in vitro and in vivo.

$H S$ activates the secretory function of $\mathrm{KCs}$. In order to determine whether HS is responsible for altering the secretory function of KCs, the concentrations of IFN- $\gamma$, IL- $1 \beta$, TNF- $\alpha$ and MIP- $1 \alpha$ in the medium of the cultured KCs were measured in each group. As presented in Fig. 2A-D, the concentrations of IFN- $\gamma$, IL-1 $\beta$, TNF- $\alpha$ and MIP-1 $\alpha$, respectively, increased during the time course $(\mathrm{P}<0.05)$. The present results demonstrate that high temperatures strongly promote the secretion of inflammatory factors from KCs. 

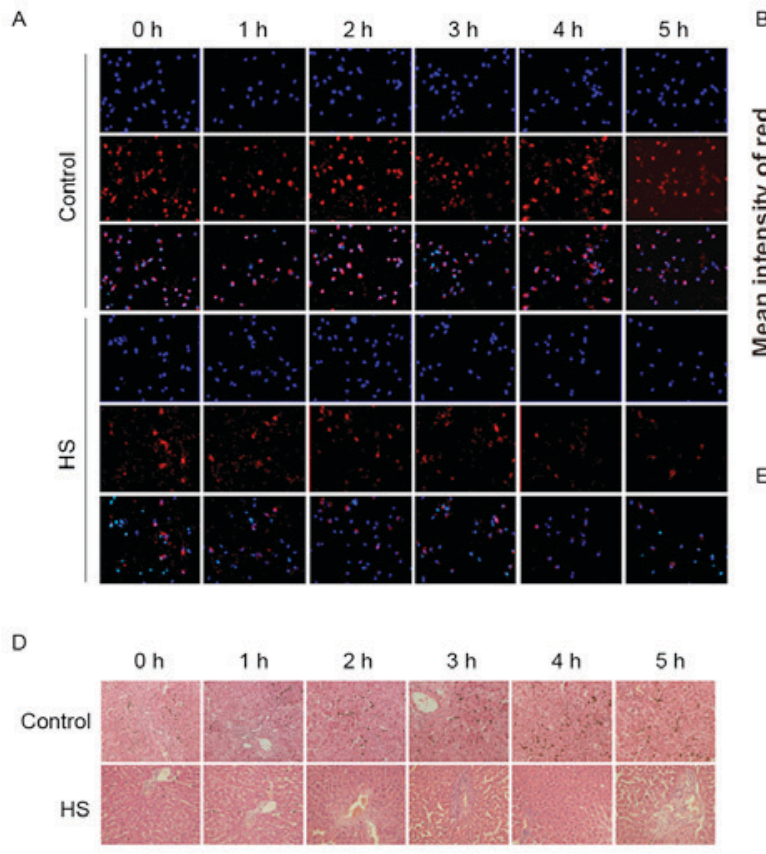
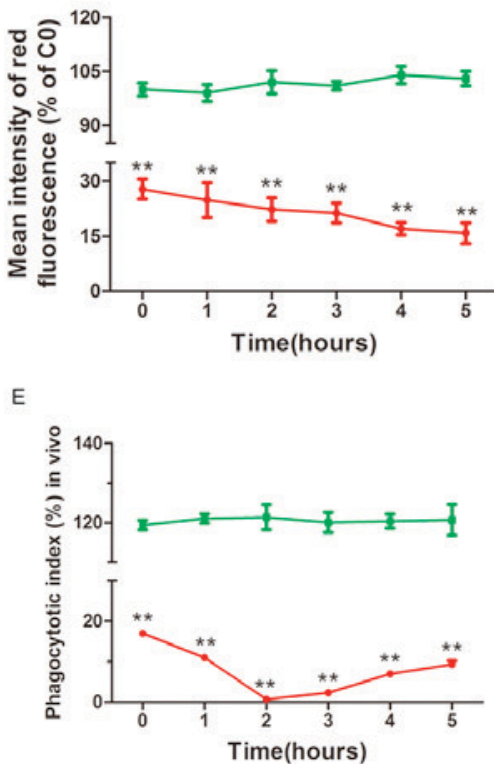
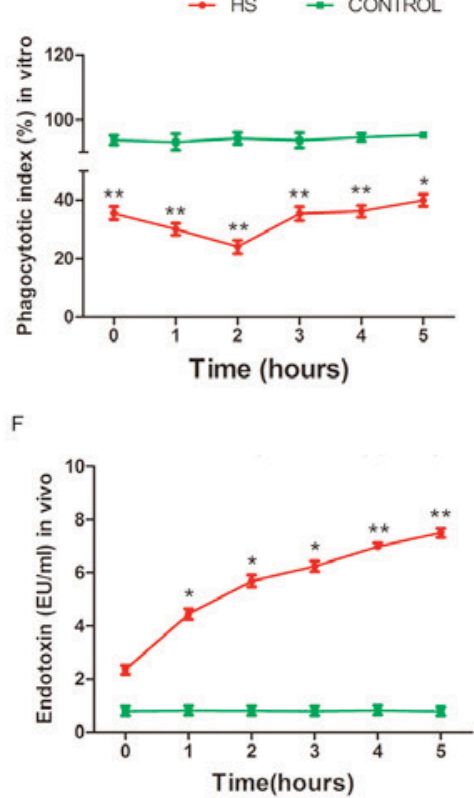

Figure 1. HS inhibits the phagocytic activity of KCs. KCs were prepared following HS for 1-5 h. (A) Latex beads (red) were applied to measure the phagocytic function of KCs, and blue fluorescence represents the nucleus. (B) The red fluorescence intensity in KCs was decreased following HS compared with that of the control cells. (C) The pHrodo Escherichia coli BioParticles Phagocytosis kit for Flow Cytometry was used to detect the phagocytic activity of the cultured rat $\mathrm{KCs}$, and the phagocytic index was decreased following HS compared with that of the control cells. (D) Subsequent to the induction of HS for 1-5 h following India ink instillation, the liver was processed and stained with eosin. The carbon consumption of KCs was observed microscopically. (E) Orbital blood was collected following the injection of diluted India ink, and the phagocytic index $\mathrm{K}$ value was calculated subsequent to the absorbance being measured. (F) The lipopolysaccharide concentration in the blood serum was detected using a ToxinSensor Chromogenic LAL Endotoxin Assay kit and these levels increased following HS. ${ }^{*} \mathrm{P}<0.05,{ }^{* *} \mathrm{P}<0.01$ vs. control. KCs, Kupffer cells; HS, heat stroke.
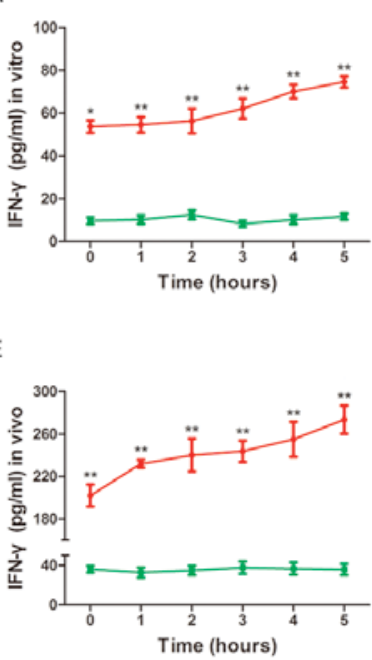

B
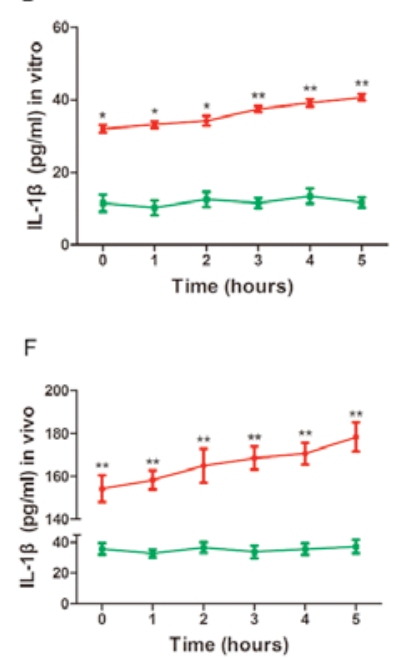
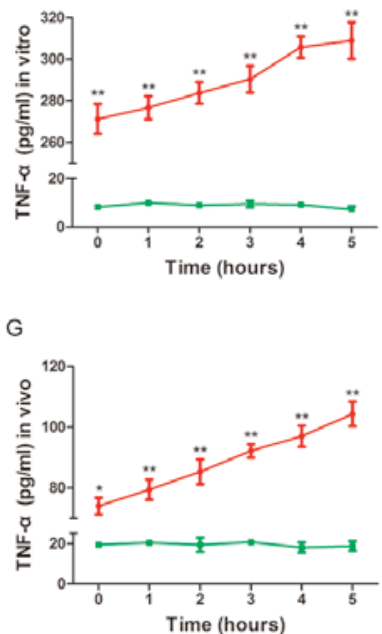

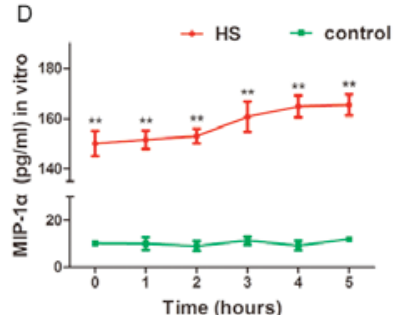

H

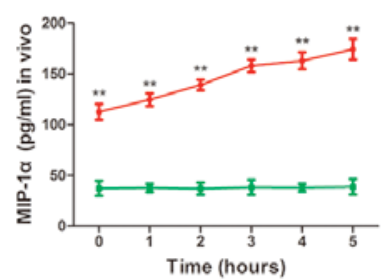

Figure 2. HS activates the secretory function of KCs. KCs and peripheral blood were prepared following HS for 1-5 h. (A) IFN- $\gamma$, (B) IL-1 $1 \beta$, (C) TNF- $\alpha$ and (D) MIP-1 $\alpha$ concentrations in the medium of cultured KCs and (E) IFN- $\gamma$, (F) IL-1 $\beta$, (G) TNF- $\alpha$ and (H) MIP-1 $\alpha$ in the peripheral blood, were detected using an ELISA in the HS and control groups. IFN- $\gamma$, IL-1 $\beta$, TNF- $\alpha$ and MIP- $1 \alpha$ concentrations increased in the HS group compared with the control group in vitro and in vivo. ${ }^{*} \mathrm{P}<0.05,{ }^{* *} \mathrm{P}<0.01$ vs. control. HS, heat stroke; KCs, Kupffer cells; LPS, lipopolysaccharide; MIP-1 $\alpha$, macrophage inflammatory protein $1 \alpha$; IFN, interferon; IL, interleukin; TNF, tumor necrosis factor.

In order to estimate the secretory function of $\mathrm{KCs}$ in vivo and further confirm that high temperatures influence the secretory function of KCs, the concentrations of IFN- $\gamma$, IL-1 $\beta$, TNF- $\alpha$ and MIP-1 $\alpha$ in the peripheral blood were measured in each group. The results of the in vivo assays were similar to those obtained in vitro. The concentrations of IFN- $\gamma$, IL- $1 \beta$, TNF- $\alpha$ and MIP- $1 \alpha$ increased during the time course, as presented in Fig. $2 \mathrm{E}-\mathrm{H}$, respectively $(\mathrm{P}<0.05)$. The present results suggest that high temperatures markedly increased the secretory functions of KCs, which promotes a severe inflammatory response with the occurrence of $\mathrm{HS}$. 


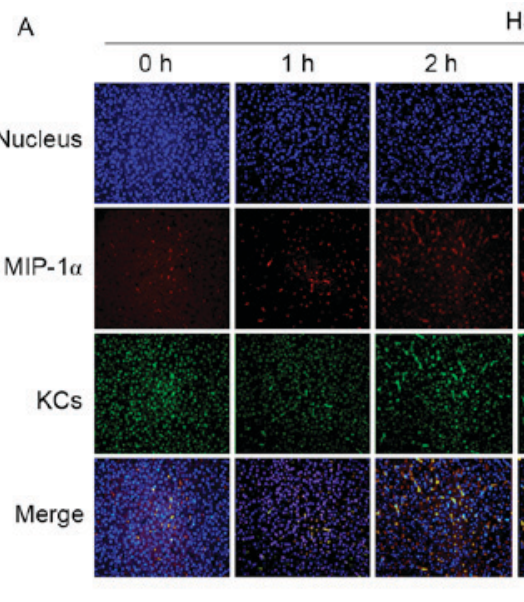

B

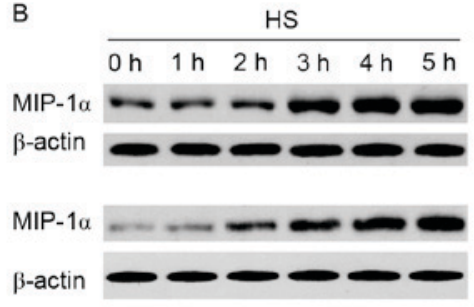

D

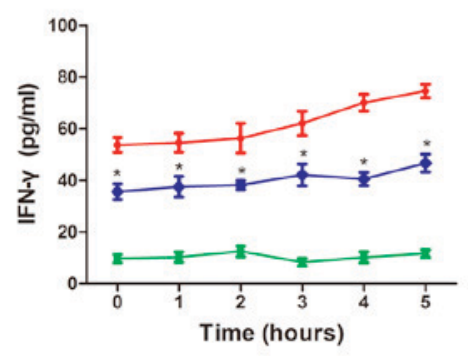

HS
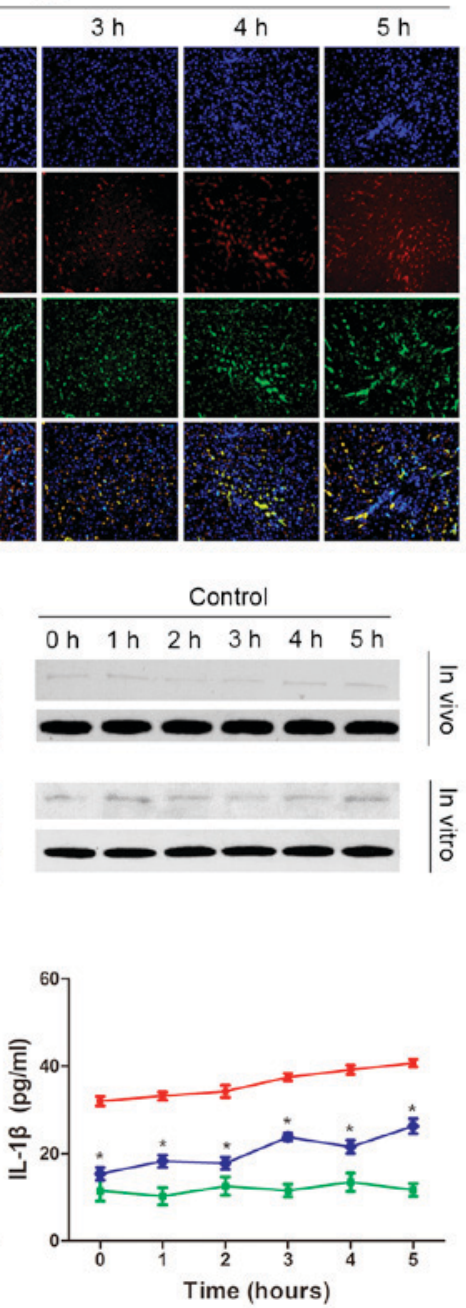

Control

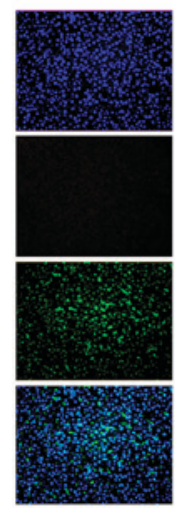

C

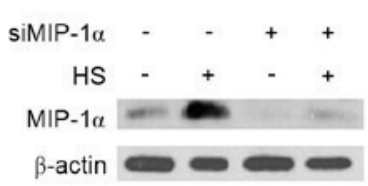

$\rightarrow$ HS $\rightarrow$ control $\rightarrow$ HS+simip-1a

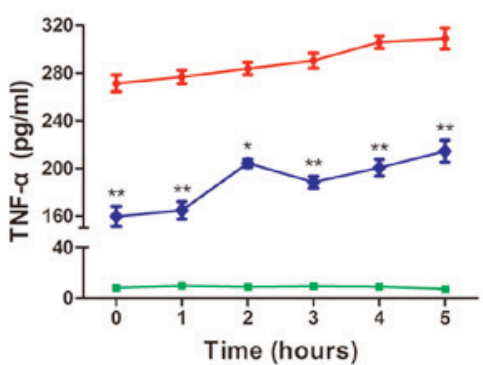

Figure 3. Expression of MIP-1 $\alpha$ positively regulates KC inflammatory function in HS. (A) The livers of the experimental animals were prepared following the induction of HS for 1-5 h. MIP-1 $\alpha$ (red) expression in the KCs (green) detected by immunofluorescence revealed an increase in expression during the time course in the HS group compared with the control group. (B) KCs and liver extracts were prepared following the induction of HS for 1-5 h. The amount of MIP-1 $\alpha$ that was up-regulated in vitro and in vivo was investigated by western blot analysis. (C) MIP-1 $\alpha$ expression was reduced using specifically targeted siRNAs in cultured KCs transfected using Lipofectamine ${ }^{\circledR} 2000$. (D) IFN- $\gamma$, IL-1 $\beta$, and TNF- $\alpha$ concentration in the medium of cultured KCs decreased in the MIP-1 $\alpha$-silenced HS group compared with the HS group detected by ELISA. ${ }^{*} \mathrm{P}<0.05,{ }^{* *} \mathrm{P}<0.01$ vs. HS group. HS, heat stroke; KCs, Kupffer cells; MIP-1 $\alpha$, macrophage inflammatory protein $1 \alpha$; IFN, interferon; IL, interleukin; TNF, tumor necrosis factor; siRNA, short interfering RNA.

Expression of MIP-1 $\alpha$ positively regulates the inflammatory function of KCs in HS. Since MIP-1 $\alpha$ concentrations increased in blood and the culture medium in the HS models, MIP-1 $\alpha$ expression in KCs was assessed in vitro and in vivo. MIP-1 $\alpha$ expression in the $\mathrm{KCs}$ of the rat livers increased gradually at the beginning of HS compared with MIP-1 $1 \alpha$ expression in the control group (Fig. 3A); additionally, MIP-1 $\alpha$ protein levels increased in the cultured liver cells and liver tissues (Fig. 3B). By silencing MIP-1 $\alpha$ in the cultured KCs (Fig. 3C), the concentrations of IFN- $\gamma$, IL- $1 \beta$ and TNF- $\alpha$ secreted by the KCs were decreased during HS compared with the HS group (Fig. 3D).

Phosphorylation of JNK increases during HS. The JNK signaling pathway may be activated in trauma, stress, and ischemia-reperfusion. In order to determine whether the JNK signaling pathway is activated by KCs in the HS model, p-JNK was assessed. As presented in Fig. 4A, the red fluorescence demonstrates that JNK phosphorylation in KCs and other liver cells increased during the time course following HS compared with the control group. As presented in Fig. 4B and C, p-JNK protein levels increased during the time course following HS compared with the control group. The present results indicate that JNK is activated during HS.

$J N K$ signaling pathway is involved in MIP-1 $\alpha$-mediated regulation of $K C$ function in $H S$. In order to explore whether the JNK signaling pathway is associated with the regulation of KC phagocytic and secretory function during HS, the JNK inhibitor sp600125 was used in the experiments with cultured KCs. The latex beads and pHrodo E. coli BioParticles Phagocytosis kit for Flow Cytometry analysis demonstrated that JNK inhibition improved the phagocytic activity of the KCs (Fig. 5A-C), whereas the detection of the inflammatory factors in the culture medium revealed that $\mathrm{KC}$ secretory function was reduced compared with the HS group (Fig. 5D-F). Secreted MIP-1 $\alpha$ levels in the KC medium were decreased via JNK inhibition. In addition, MIP-1 $\alpha$ levels in KC total protein 

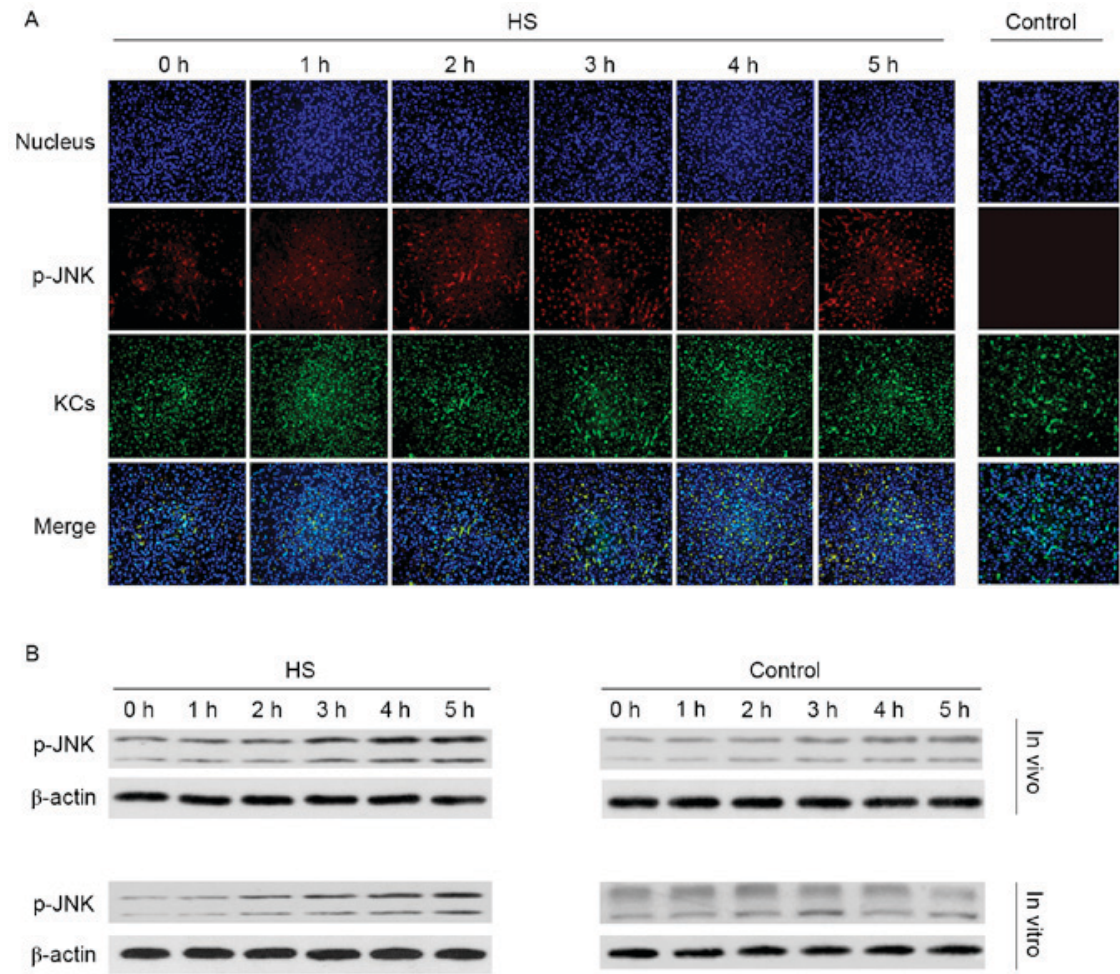

Figure 4. JNK phosphorylation increases with HS. (A) The livers of experimental animals were prepared following HS induction for 1-5 h. JNK phosphorylation (red) in KCs (green), as detected by immunofluorescence, revealed increased levels during the time course in the HS group compared with the control group. (B) KCs and liver extracts were prepared following HS induction for 1-5 h. JNK phosphorylation increased in vitro and in vivo as assessed by western blot analysis. JNK, c-Jun-N-terminal kinase; HS, heat stroke; KCs, Kupffer cells.

A

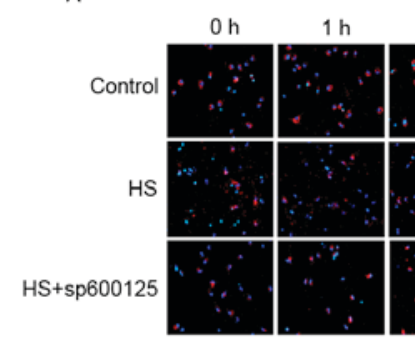

D

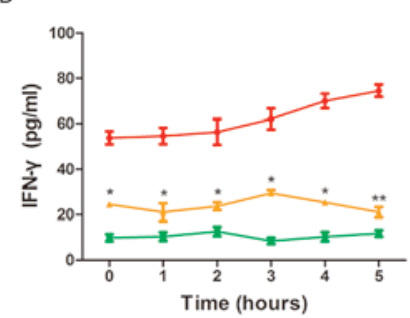

H

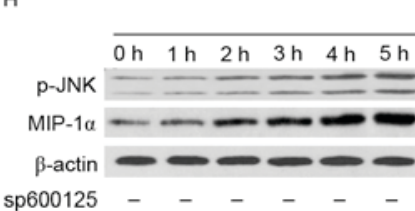

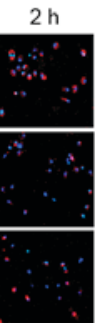
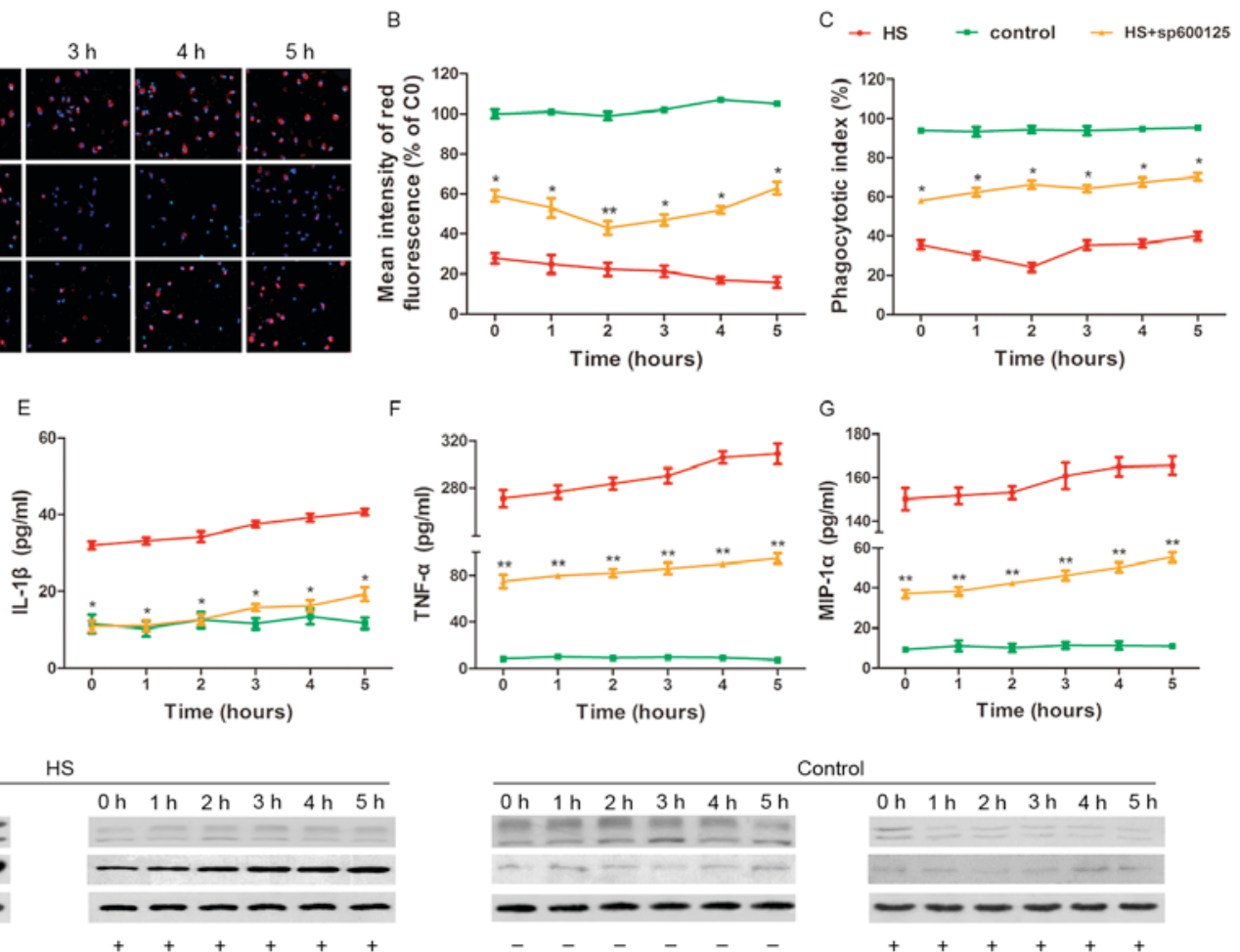
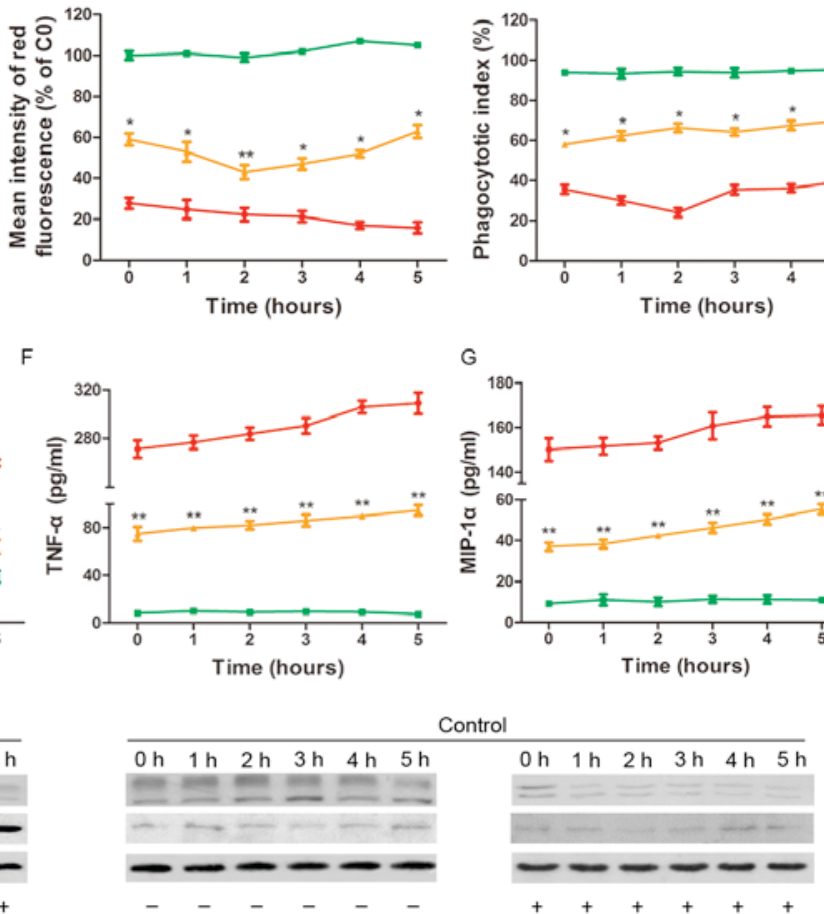

Figure 5. JNK signaling pathway is involved in MIP-1 $\alpha$-mediated regulation of KC function in HS. (A) Latex beads (red) were applied to measure the phagocytic function of KCs, and blue fluorescence represents the nucleus. (B) With the sp600125 treatment, the red fluorescence intensity in the KCs significantly increased following HS compared with the cells without sp600125 treatment. (C) The phagocytic index increased following sp600125 treatment compared with HS cells without the sp600125 treatment. (D-G) IFN- $\gamma$, IL-1 $\beta$, TNF- $\alpha$ and MIP-1 $\alpha$ concentrations in the medium of cultured KCs decreased following sp600125 treatment measured by ELISA, compared with HS cells without sp600125 treatment. (H) Following HS, MIP-1 $\alpha$ levels decreased in vitro subsequent to sp600125 pretreatment, as detected by western blot analysis, compared with cells not exposed to pretreatment. ${ }^{*} \mathrm{P}<0.05,{ }^{* * *} \mathrm{P}<0.01$ vs. HS group. MIP-1 $\alpha$, macrophage inflammatory protein $1 \alpha$; IFN, interferon; IL, interleukin; TNF, tumor necrosis factor; KCs, Kupffer cells; JNK, c-Jun-N-terminal kinase; HS, heat stroke. 
extractions were reduced compared with the HS group. The present results suggest that JNK is involved in the regulation of $\mathrm{KC}$ function.

\section{Discussion}

In the present study, it was demonstrated that the phagocytic activity of KCs is reduced and the secretory function of KCs is increased in vitro and in vivo following HS. Additionally, the JNK signaling pathway is potentially responsible for MIP-1 $\alpha$-mediated regulation of $\mathrm{KC}$ function in the $\mathrm{HS}$ cellular model.

$\mathrm{KCs}$ are resident macrophages that serve important roles in liver processes, including regeneration, fibrogenesis, inflammation and necrosis (11). The inflammatory cytokines produced by activated KCs are involved in the pathogenesis of various types of liver injury, including viral hepatitis (25), alcoholic liver disease (26) and ischemia-reperfusion injury (27). Previous studies have demonstrated that the secretory function of KCs changes in several conditions, including hyperpyrexia, and that the inhibition of cytokine secretion by KCs leads to the reduction of liver injury (5). In the present study, an acute increase in the secretion of inflammatory factors by $\mathrm{KCs}$ subsequent to HS being observed, indicating that HS activates the $\mathrm{KC}$-mediated inflammation response.

$\mathrm{KCs}$ eliminate and decompose various exogenous and endogenous substances, including gut-derived endotoxins in the liver, which is an essential regulatory function. LPS, which is located on the outer wall of gram-negative bacteria, is predominantly eliminated by $\mathrm{KCs}$ in the liver (28). KC activation by LPS causes the release of IL-1, IL-6, TNF- $\alpha$ and other inflammatory cytokines and triggers additional inflammatory processes (29). Elevated levels of endotoxin (LPS) delivered to the liver via portal blood may cause liver injury (30). In the present study, a decrease in the phagocytic activity of $\mathrm{KCs}$ following HS was noted, and increased LPS levels indicated that the ability of KCs to decompose LPS was weakened.

As the phagocytic activity of KCs decreases while the secretory function increases during HS to promote inflammatory responses, and since MIP-1 $\alpha$ is a pro-inflammatory factor in macrophages, MIP-1 $\alpha$ was the focus of the present study. MIP- $1 \alpha$ is a member of the cysteine chemokine subfamily and is involved in the regulation of cell-mediated immunity (31). MIP-1 $\alpha$ attracts and stimulates basophils to secrete pro-inflammatory substances (32). Previous studies have demonstrated MIP-1 $\alpha$ protein and mRNA overexpression in KCs following liver ischemia-reperfusion injury (19). As little is known regarding MIP-1 $\alpha$ expression in heat-stressed conditions, a model of HS was established in order to investigate alterations in MIP-1 $\alpha$ expression in $\mathrm{KCs}$, and it was identified that MIP-1 $\alpha$ expression increased gradually following HS onset. However, MIP-1 $\alpha$ silencing in KCs resulted in reduced levels of inflammatory cytokine secretion by KCs subsequent to HS compared with the HS group.

In order to investigate the mechanism of MIP- $1 \alpha$-associated translation of the production of pro-inflammatory cytokines by $\mathrm{KCs}$ in $\mathrm{HS}$, the stress-associated nuclear import pathways, including JNK signaling, were examined. The JNK signaling pathway is reported to be associated with the development of liver inflammation and mammalian TNF- $\alpha$ signaling $(20,33)$. JNK serves a major role in cell responses, inflammation, apoptosis and heat stress, particularly in KCs $(16,34,35)$. However, an association between the function of KCs and JNK has not been established. Consistent with these observations, a time-dependent activation of JNK phosphorylation was demonstrated 1-5 h following HS. Following inhibition of JNK phosphorylation by sp600125 in KCs in vitro and in vivo, decreased amounts of inflammatory cytokines and MIP- $1 \alpha$ were produced from activated $\mathrm{KCs}$, whereas increased phagocytic activation of KCs was detected following HS compared with that in the HS group without inhibition.

In the present study, all of the experiments were undertaken with animal and cell models, which provide a powerful tool for HS studies. However, the results obtained with the models may not be consistent with those obtained from studies in humans, therefore further clinical studies are required.

In conclusion, the present study indicates that $\mathrm{KC}$ function alters in HS-induced injury. Furthermore, it emphasizes that HS enriches the expression of MIP- $1 \alpha$ in KCs and the phosphorylation of JNK, and that JNK signaling is required for the HS response.

\section{Acknowledgements}

The present study was supported by the China Postdoctoral Science Foundation (grant no. 2015M572816).

\section{References}

1. Widodo D: Heat stroke. Acta Med Indones 37: 39-42, 2005.

2. Keatinge WR: Death in heat waves. BMJ 327: 512-513, 2003.

3. Misset B, De Jonghe B, Bastuji-Garin S, Gattolliat O, Boughrara E, Annane D, Hausfater P, Garrouste-Orgeas M and Carlet J: Mortality of patients with heatstroke admitted to intensive care units during the 2003 heat wave in France: A national multiple-center risk-factor study. Crit Care Med 34: 1087-1092, 2006.

4. Li L, Gu Z, Liu Z and Su L: The effect of reactive oxygen species regulation of expression of $\mathrm{Bcl}-2$ and Bax in apoptosis of human umbilical vein endothelial cell induced by heat stress. Zhonghua Wei Zhong Bing Ji Jiu Yi Xue 26: 458-463, 2014 (In Chinese).

5. Chen Y, Tong H, Zhang X, Tang L, Pan Z, Liu Z, Duan P and Su L: Xuebijing injection alleviates liver injury by inhibiting secretory function of Kupffer cells in heat stroke rats. J Tradit Chin Med 33: 243-249, 2013.

6. Lu KC, Wang JY, Lin SH, Chu P and Lin YF: Role of circulating cytokines and chemokines in exertional heatstroke. Crit Care Med 32: 399-403, 2004.

7. Rodriguez-Fernandez M, Grosman B, Yuraszeck TM, Helwig BG, Leon LR and Doyle FJ III: Modeling the intra- and extracellular cytokine signaling pathway under heat stroke in the liver. PLoS One 8: e73393, 2013.

8. Hall DM, Xu L, Drake VJ, Oberley LW, Oberley TD, Moseley PL and Kregel KC: Aging reduces adaptive capacity and stress protein expression in the liver after heat stress. J Appl Physiol (1985) 89: 749-759, 2000.

9. Jirillo E, Caccavo D, Magrone T, Piccigallo E, Amati L, Lembo A, Kalis C and Gumenscheimer M: The role of the liver in the response to LPS: Experimental and clinical findings. J Endotoxin Res 8: 319-327, 2002.

10. Fukuda T, Mogami A, Tanaka H, Yoshikawa T, Hisadome M and Komatsu H: Y-40138, a multiple cytokine production modulator, protects against D-galactosamine and lipopolysaccharide-induced hepatitis. Life Sci 79: 822-827, 2006.

11. Kolios G, Valatas V and Kouroumalis E: Role of Kupffer cells in the pathogenesis of liver disease. World J Gastroenterol 12: 7413-7420, 2006.

12. Liu LM, Liang DY, Ye CG, Tu WJ and Zhu T: The UII/UT system mediates upregulation of proinflammatory cytokines through p38 MAPK and NF-kappaB pathways in LPS-stimulated Kupffer cells. PLoS One 10: e0121383, 2015. 
13. Vollmar B and Menger MD: The hepatic microcirculation: Mechanistic contributions and therapeutic targets in liver injury and repair. Physiol Rev 89: 1269-1339, 2009.

14. Kono H, Fujii H, Asakawa M, Yamamoto M, Maki A, Matsuda M, Rusyn I and Matsumoto Y: Functional heterogeneity of the kupffer cell population is involved in the mechanism of gadolinium chloride in rats administered endotoxin. J Surg Res 106: 179-187, 2002

15. Fahey TJ III, Tracey KJ, Tekamp-Olson P, Cousens LS, Jones WG, Shires GT, Cerami A and Sherry B: Macrophage inflammatory protein 1 modulates macrophage function. J Immunol 148: 2764-2769, 1992.

16. Giribaldi G, Prato M, Ulliers D, Gallo V, Schwarzer E, Akide-Ndunge OB, Valente E, Saviozzi S, Calogero RA and Arese P: Involvement of inflammatory chemokines in survival of human monocytes fed with malarial pigment. Infect Immun 78: 4912-4921, 2010

17. Hsieh CH, Frink M, Hsieh YC, Kan WH, Hsu JT, Schwacha MG, Choudhry MA and Chaudry IH: The role of MIP-1 alpha in the development of systemic inflammatory response and organ injury following trauma hemorrhage. J Immunol 181: 2806-2812, 2008

18. Nishi T, Maier CM, Hayashi T, Saito A and Chan PH: Superoxide dismutase 1 overexpression reduces MCP-1 and MIP-1 alpha expression after transient focal cerebral ischemia. J Cereb Blood Flow Metab 25: 1312-1324, 2005.

19. Ma W, Wang ZR, Shi L and Yuan Y: Expression of macrophage inflammatory protein-1alpha in Kupffer cells following liver ischemia or reperfusion injury in rats. World J Gastroenterol 12: 3854-3858, 2006.

20. Seki E, Brenner DA and Karin M: A liver full of JNK: Signaling in regulation of cell function and disease pathogenesis, and clinical approaches. Gastroenterology 143: 307-320, 2012.

21. Mukhopadhyay P, Rajesh M, Horváth B, Bátkai S, Park O Tanchian G, Gao RY, Patel V, Wink DA, Liaudet L, et al: Cannabidiol protects against hepatic ischemia/reperfusion injury by attenuating inflammatory signaling and response, oxidative/nitrative stress, and cell death. Free Radic Biol Med 50: 1368-1381, 2011.

22. Ikezumi Y, Hurst L, Atkins RC and Nikolic-Paterson DJ: Macrophage-mediated renal injury is dependent on signaling via the JNK pathway. J Am Soc Nephrol 15: 1775-1784, 2004.
23. Chan ED, Winston BW, Jarpe MB, Wynes MW and Riches DW: Preferential activation of the p46 isoform of JNK/SAPK in mouse macrophages by TNF alpha. Proc Natl Acad Sci USA 94: 13169-13174, 1997.

24. Liu JW: The effect of endotoxin tolerance on the Galn/LPS induced MAPK and STAT signal transduction. Shanxi Medical University, 2006

25. Adams DH and Hubscher SG: Systemic viral infections and collateral damage in the liver. Am J Pathol 168: 1057-1059, 2006.

26. Gregory SH and Wing EJ: Neutrophil-Kupffer cell interaction: A critical component of host defenses to systemic bacterial infections. J Leukoc Biol 72: 239-248, 2002.

27. Su GL: Lipopolysaccharides in liver injury: Molecular mechanisms of Kupffer cell activation. Am J Physiol Gastrointest Liver Physiol 283: G256-G265, 2002.

28. Diesen DL and Kuo PC: Nitric oxide and redox regulation in the liver: Part II. Redox biology in pathologic hepatocytes and implications for intervention. J Surg Res 167: 96-112, 2011.

29. Tukov FF, Luyendyk JP, Ganey PE and Roth RA: The role of tumor necrosis factor alpha in lipopolysaccharide/ranitidine-induced inflammatory liver injury. Toxicol Sci 100: 267-280, 2007.

30. Thakur V, Pritchard MT, McMullen MR, Wang Q and Nagy LE: Chronic ethanol feeding increases activation of NADPH oxidase by lipopolysaccharide in rat Kupffer cells: Role of increased reactive oxygen in LPS-stimulated ERK1/2 activation and TNF-alpha production. J Leukoc Biol 79: 1348-1356, 2006.

31. Matsukawa A, Hogaboam CM, Lukacs NW and Kunkel SL: Chemokines and innate immunity. Rev Immunogenet 2: 339-358, 2000.

32. Kaplan AP, Kuna P and Reddigari SR: Chemokines and the allergic response. Exp Dermatol 4: 260-265, 1995.

33. Baud V and Karin M: Signal transduction by tumor necrosis factor and its relatives. Trends Cell Biol 11: 372-377, 2001

34. Shen J, Sakaida I, Uchida K, Terai S and Okita K: Leptin enhances TNF-alpha production via p38 and JNK MAPK in LPS-stimulated Kupffer cells. Life Sci 77: 1502-1515, 2005.

35. Gonda RL, Garlena RA and Stronach B: Drosophila heat shock response requires the JNK pathway and phosphorylation of mixed lineage kinase at a conserved serine-proline motif. PLoS One 7: e42369, 2012. 